\title{
Alternative sintering methods compared to conventional thermal sintering for inkjet printed silver nanoparticle ink
}

\author{
Juha Niittynen a,*, Robert Abbel ${ }^{b}$, Matti Mäntysalo a, Jolke Perelaer ${ }^{c, d}$, Ulrich S. Schubert ${ }^{c, d}$, Donald Lupo a \\ a Department of Electronics and Communications Engineering, Tampere University of Technology, Korkeakoulunkatu 3, 33720 Tampere, Finland \\ b Holst Centre, High Tech Campus 31, 5656 AE Eindhoven, The Netherlands \\ c Laboratory of Organic and Macromolecular Chemistry (IOMC), Friedrich-Schiller-University Jena, Humboldtstrasse 10, D-07743 Jena, Germany \\ d Jena Center for Soft Matter (JCSM), Friedrich-Schiller-University Jena, Humboldtstrasse 10, D-07743 Jena, Germany
}

\section{A R T I C L E I N F O}

\section{Article history:}

Received 22 January 2013

Received in revised form 5 September 2013

Accepted 3 February 2014

Available online 8 February 2014

\section{Keywords:}

Inkjet

Sintering

Plasma

Photonic

Laser

Thermal

\begin{abstract}
A B S T R A C T
In this contribution several alternative sintering methods are compared to traditional thermal sintering as high temperature and long process time of thermal sintering are increasing the costs of inkjet-printing and preventing the use of this technology in large scale manufacturing. Alternative sintering techniques are evaluated based on the electrical and mechanical performance they enable on inkjet-printed structures as well as their potential feasibility for large scale manufacturing. Photonic sintering was identified as the most promising alternative to thermal sintering.
\end{abstract}

(c) 2014 Elsevier B.V. All rights reserved.

\section{Introduction}

Flexible electronics combine bendable substrates with inorganic materials by means of printing technologies, enabling light-weight and extremely costs-efficient electronic systems [1]. A highly viable and innovative fusion of three technological areas - microelectronics, chemistry and printing - is foreseen in the near future [2].

For example, radiofrequency identification tags $[2,3]$ and various flexible electronic devices, including batteries [2,4], (organic) photovoltaics [5] as well as logic and memory components [6], are among the interesting and possible application areas for inkjet printed electronics. Inkjet printing has been shown to be suitable for electronic miniaturization and rapid research and development prototyping $[7,8]$. Furthermore, inkjet printing represents a digital "drop-on-demand" manufacturing technique that delivers expensive materials on demand, which reduces the material waste during the manufacturing process. Furthermore, the process efficiency is improved as well as the environmental stress of the manufacturing process is reduced [9].

Inkjet printing is currently moving from the lab-scale to a manufacturing scale, which demands a significantly decreased price as well as increased process output in order to become competitive towards conventional manufacturing methods [10,11].

An important requirement for microelectronic devices is the conductive structures, which makes metals the most obvious choice due

\footnotetext{
* Corresponding author. Tel.: + 35840849 0624; fax: + 35833641445 .

E-mail address: juha.niittynen@tut.fi (J. Niittynen).
}

to the superior conductivity compared to organic materials. Inkjet printing of metal containing precursor materials has been widely accepted as a suitable processing alternative for the fabrication of contacts and interconnects at relatively high speed that enables rollto-roll production [9]. The required post-printing sintering step to render the precursor materials conductive, high process temperature is usually required, which means that most of the cost-effective polymer foils, such as polyethylene terephthalate or polycarbonate (PC), which have relatively low glass transition temperature ( $\mathrm{Tg}$ ) are not compatible with traditional thermal sintering [12]. As an alternative, more expensive polymer substrates, such as polyimide or polyarylate, can be used [13]. These expensive materials obviously hamper the cost-efficiency of the production process.

Two different classes of conductive inks are commonly used for inkjet printing, either based on metal-organic decomposition complexes, or metal nanoparticles [7]. The metal nanoparticles in an ink are generally stabilized by an organic layer in order to keep the nanoparticles well-dispersed in solution. In order to gain conductivity, the organic moieties first need to be removed after the printing process. Subsequently, the particles make direct physical contact, followed by neck formation between the particles and larger particles will be formed, triggered by Ostwald ripening and the surface-to-volume reduction [14]. Upon sintering a continuous percolating network is formed throughout the printed features, resulting in electrical conductivity.

The organic stabilizer is typically removed by heat, but this process is not compatible with common polymer foils due to their high thermal 

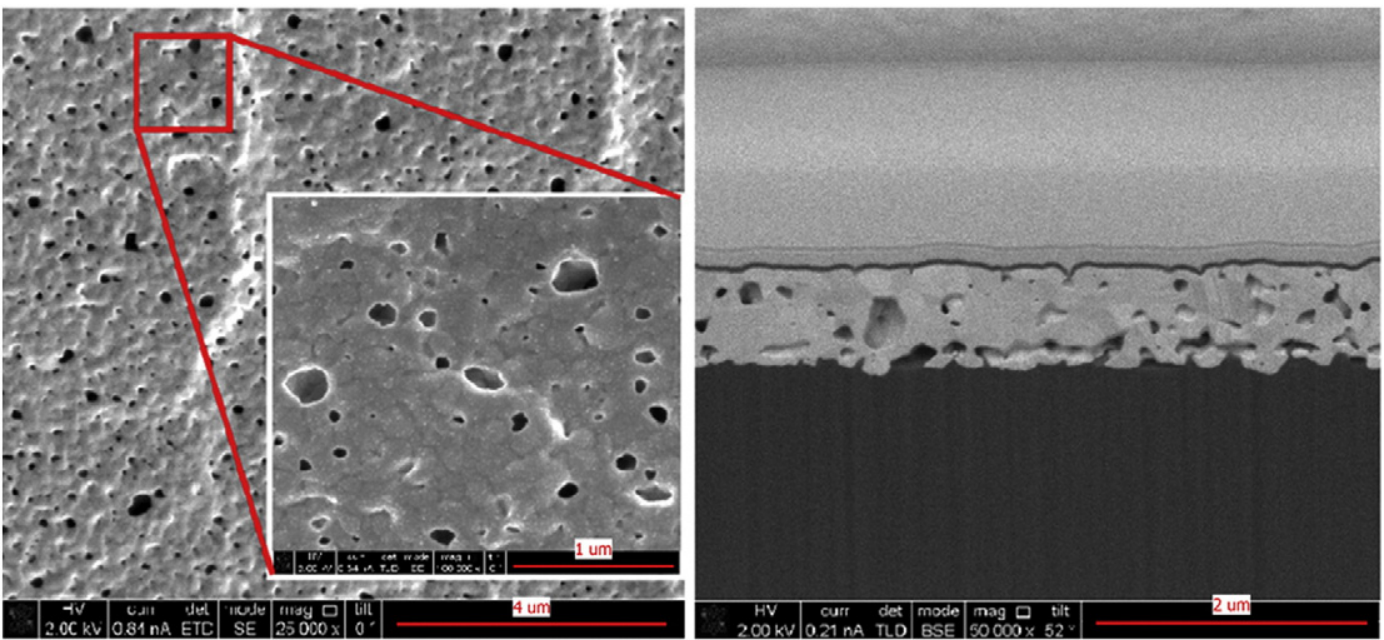

Fig. 1. Top-view and cross-section SEM images from a thermally sintered sample.

sensitivity [15]. Therefore, alternative sintering techniques are required to reduce the manufacturing costs and shorten the processing time.

In recent years, researchers have reported various methods to reduce the sintering temperatures of metal precursor inks by tailoring the organic content in the ink $[16,17]$. Instead of heating the complete sample, more selective sintering techniques were used by various groups, including laser sintering [18], low pressure argon plasma exposure [19], microwave radiation [20], electrical [21] and photonic sintering [22]. These techniques converted metal precursor inks into conductive features and excellent conductivity values up to $60 \%$ were obtained in short processing times [23].

The electrical performance of the printed and sintered features is essential for the final electronic applications; a low conductivity causes unnecessary power losses throughout the device due to increased resistances, resulting in excess heating and possibly reliability issues. Furthermore, a low conductivity can disturb high frequency conductor lines and time-critical signal lines [24-27]. A conductivity of one tenth of bulk silver material can be considered to be adequate for some lowperformance applications.

Secondly, the mechanical performance of printed and sintered features is important to evaluate, since it directly relates to the reliability of the printed structures. Poor mechanical properties lead to a decreased reliability and a shortened lifetime, in particular in the dynamic and flexible applications where mechanical stresses are more prominent than in rigid printed circuit boards [28]. The ability to endure mechanical stresses caused by the flexible action is very important as flexible devices are considered as one of the most promising applications for printable electronics [29].

It is therefore extremely important to achieve good sintering and a homogeneous microstructure as a porous microstructure means that

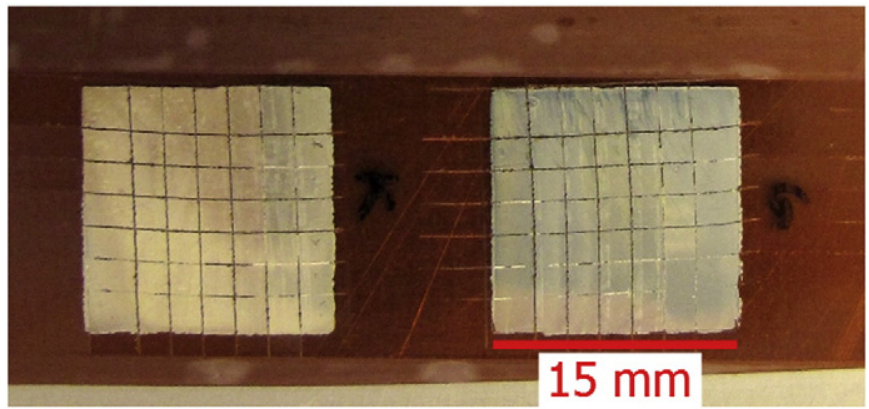

Fig. 2. Peel-off adhesion test sample for thermal sintering on polyimide. The size of the peel-off adhesion test squares is $15 \mathrm{~mm}$ by $15 \mathrm{~mm}$. the local conductivities may vary greatly even inside a single sample. Local differences in conductivity may cause "hot spots" where the current density is higher which, in turn, may cause local heating and therefore reliability issues within the structure. Non-uniform sintering is undesirable as the large-scale electronic manufacturing demands highly repeatable and reproducible fabrication processes in order to achieve the desired levels of long-term reliability.
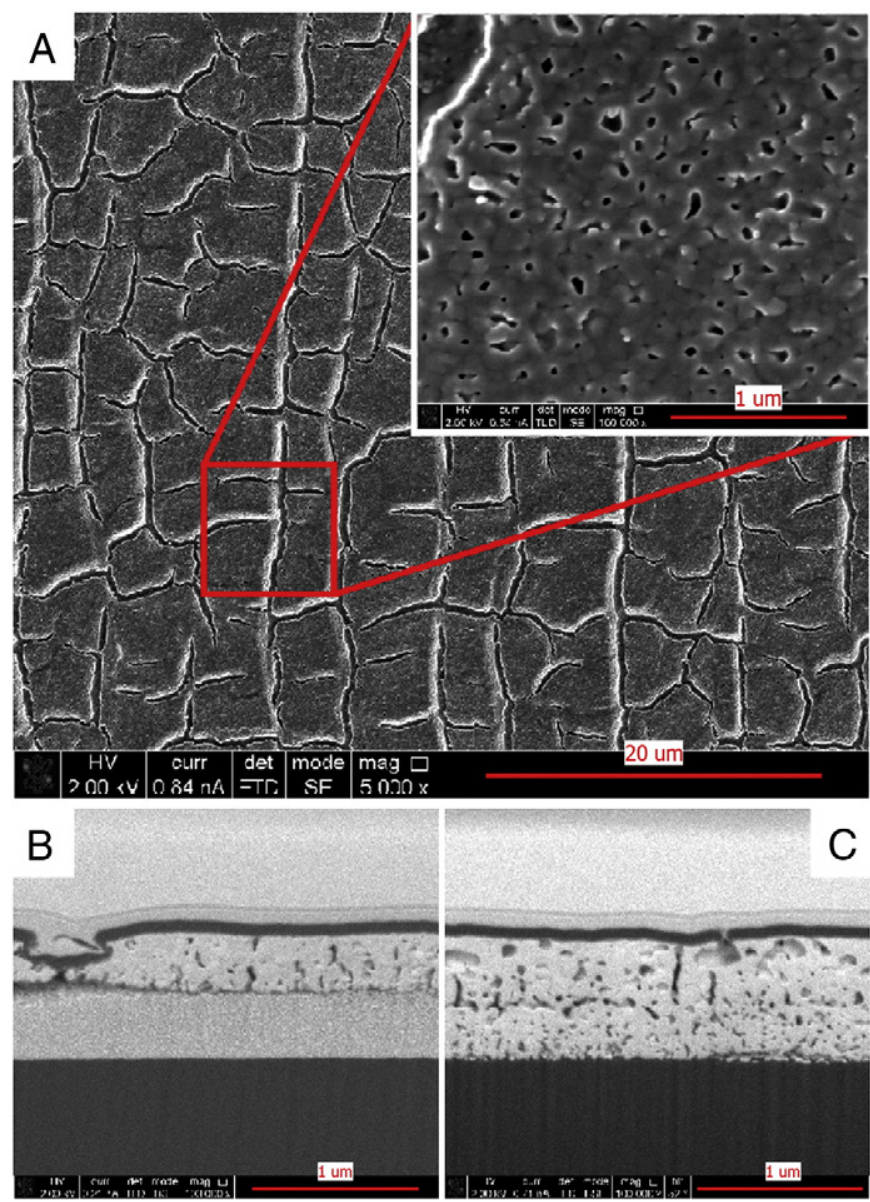

Fig. 3. Top-view (after $4 \mathrm{~h}$ ) and cross-section (after $30 \mathrm{~min}$ (A) and after $4 \mathrm{~h}$ (B)) of plasma sintering. 
Table 1

Resistivity and peel-off adhesion test results for plasma sintering.

\begin{tabular}{lll}
\hline Sintering time $(\mathrm{min})$ & Resistivity $(\mu \Omega \mathrm{cm})$ & Adhesion $($ ASTM D-3359) \\
\hline 30 & $33.3-38.8$ & 0 \\
60 & $23.3-23.8$ & 0 \\
120 & $17.5-18.8$ & 0 \\
240 & $15.0-15.7$ & 0 \\
\hline
\end{tabular}

In this contribution, three alternative sintering methods, including plasma, photonic and laser sintering and their sintering performance are investigated for an inkjet printed silver nanoparticle ink on polymer substrates. Different sintering techniques are compared by measuring the resulting resistivity as well as the adhesion to the underlying polymer substrate. As the delamination of the printed structure from the substrate is the most common failure mode for inkjet printed structures, it is justified to use the adhesion as an indicator for the overall mechanical performance of the entire structure. Electrical conductivities as well as adhesion studies have not been compared up to now in the literature for the various sintering techniques.

\section{Experimental}

\subsection{Materials}

A commercially available silver nanoparticle dispersion from Harima (NPS-J, Harima Chemicals, Japan) was inkjet printed onto transparent polycarbonate (Bayer Materials Science, Germany, $125 \mu \mathrm{m}$ thickness, $\mathrm{Tg}=148{ }^{\circ} \mathrm{C}$ ) and polyimide (PI, $3 \mathrm{M}$ Kapton $125 \mathrm{HN}$, USA, $50 \mu \mathrm{m}$ thickness, $\mathrm{Tg}>360^{\circ} \mathrm{C}$ ) substrates. Some sintering techniques require high thermal endurance and thus polyimide is used for all resistivity and adhesion test in order to provide comparable results. In addition polycarbonate substrate is also used in adhesion testing for plasma sintering in order to better observe the sintering behavior on the bottom of the printed structure. As the visual estimation of sintering progress relies mostly in color change of the silver ink the colorless polycarbonate film is a better suited substrate than orange-hued polyimide.

\subsection{Instruments}

Inkjet printing was performed using a piezoelectric Dimatix DMP 2830 system (Dimatix-Fujifilm Inc., USA), equipped with a $10 \mathrm{pL}$ cartridge (DMC-11610). Simple line structures of $15 \mathrm{~mm}$ in length and $300 \mu \mathrm{m}$ in width as well as films of $15 \mathrm{~mm}$ by $15 \mathrm{~mm}$ were used for the conductivity and adhesion tests, respectively. Drop size was about $35 \mu \mathrm{m}$ and printing resolution of 900 drops per inch was used. Prior to printing the substrates were cleaned by rinsing with isopropanol and subsequently dried with a nitrogen flow. After printing the samples were dried on the heated print plate $\left(60^{\circ} \mathrm{C}\right)$ for minimum of $15 \mathrm{~min}$ and couple of days in room conditions before sintering.

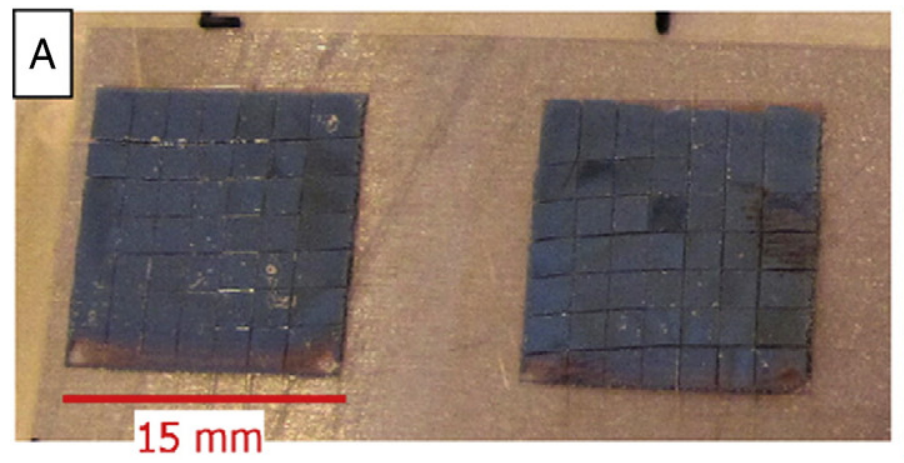

Thermal sintering was used as a reference for the alternative sintering methods and was carried out in a convection oven at $220^{\circ} \mathrm{C}$ for $1 \mathrm{~h}$, according to the material manufacturer's recommendation. Plasma sintering was performed using a low-pressure plasma instrument from Diener Electronic (Ebhausen, Germany). Argon was used as a process gas. The plasma generator operates at a frequency of $40 \mathrm{kHz}$ and the power was set to $300 \mathrm{~W}$.

Laser sintering was done utilizing an $808 \mathrm{~nm}$ laser (HLU35C10X2808-CB, Lissotschenko Mikrooptik GmbH, Germany) that has a $35 \mathrm{~W}$ nominal power output. The size of the rectangular spot is approximately $3 \mathrm{~mm}$ by $500 \mu \mathrm{m}$. A custom made control system was built for the laser sintering purposes utilizing the digitally controlled laser power unit and stage control. This sintering setup allows a fully digitally controlled sintering process that can be customized separately for each device architecture.

Photonic flash sintering was carried out in a custom-made apparatus, the details of which are disclosed in reference [30]. The inner surface of the elliptic reflector was covered by a highly reflective foil (reflectivity over 98\%). The Xenon flash lamp (XOP-50, Philips, The Netherlands) has a maximum power of $1000 \mathrm{~W}$, an emission spectrum ranging from 350 to $900 \mathrm{~nm}$ and is able to produce $10 \mathrm{~ms}$ pulses up to a frequency of $50 \mathrm{~Hz}$. The sample is placed at a fixed position and the lamp focus area (on which the intensity is above $80 \%$ of the maximum) is approximately $5 \mathrm{~mm}$ by $5 \mathrm{~cm}$.

\subsection{Characterization}

Scanning electron microscopy (SEM) images were taken using a FEG E-SEM XL30 (Philips, The Netherlands). Cross-section SEM images are acquired using a Philips Nova 200 Nanolab SDB focused ion beam (FIB) cutting equipment. The resistance was measured using a multimeter (Voltcraft Plus VC 960, Conrad, Germany).

The adhesion between the inkjet printed structure and the substrate was analyzed using the ASTM D3359-B standard [31]. The inkjet printed squares on polymer substrates were scratched utilizing a sharp tool in a controlled manner and divided into 49 individual squares. A special adhesion tape was then pressed on top of the structure and manually torn off. The adhesion category of a printed structure is defined based on the pattern that is left on the substrate after the peel-off and categorized according to the classes described in the ASTM standard.

\section{Results and discussion}

\subsection{Thermal sintering}

The first sintering technique that is discussed here, is thermal sintering, as it is the most commonly used sintering method for inkjet printed nanoparticle inks. This technique will be used as a reference for the three alternative sintering methods that are described in the next sections.

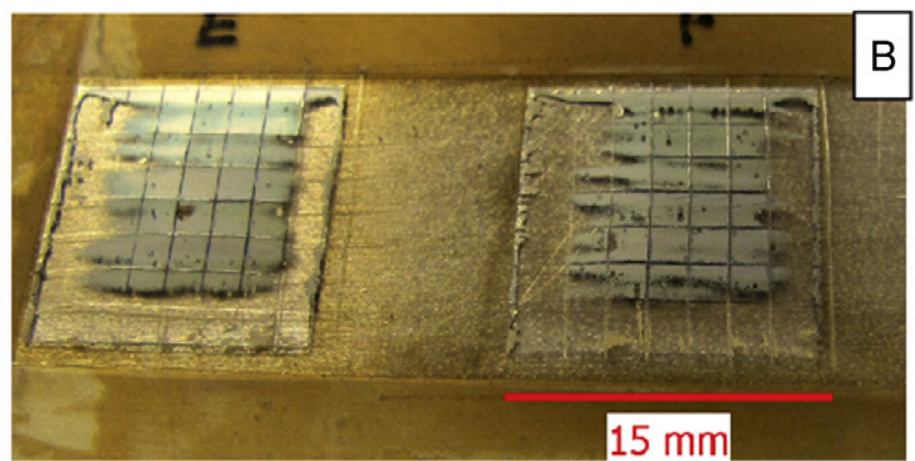

Fig. 4. Adhesion test results after $30 \mathrm{~min}(\mathrm{~A})$ and $4 \mathrm{~h}$ (B) of plasma sintering. The size of the peel-off adhesion test squares is $15 \mathrm{~mm}$ by $15 \mathrm{~mm}$. 
Table 2

Resistivity and peel-off adhesion test results for laser sintering.

\begin{tabular}{llll}
\hline $\begin{array}{l}\text { Sintering power } \\
(\mathrm{W})\end{array}$ & $\begin{array}{l}\text { Scanning speed } \\
(\mathrm{mm} / \mathrm{s})\end{array}$ & $\begin{array}{l}\text { Resistivity } \\
(\mu \Omega \mathrm{cm})\end{array}$ & $\begin{array}{l}\text { Adhesion } \\
(\text { ASTM D-3359) }\end{array}$ \\
\hline 38 & 100 & $4.6-7.2$ & 0 \\
38 & 80 & $4.6-6.2$ & 0 \\
34 & 80 & $5.7-8.1$ & 0 \\
29 & 80 & $5.8-8.9$ & 0 \\
24 & 80 & $8.4-9.0$ & 0 \\
\hline
\end{tabular}

Inkjet printing was here performed on polyimide substrates due to the thermal endurance caused by the high processing temperature. Fig. 1 shows top-view and cross-sectional scanning electron microscopy images of thermally sintering nanoparticles at $220^{\circ} \mathrm{C}$ for $1 \mathrm{~h}$. It can be seen that the initially small silver nanoparticles have merged into larger clusters and a continuous percolating network of conductive material has formed, confirmed by a low resistivity of $3.6 \mu \Omega \mathrm{cm}$, which is $2.2 \times$ more than the bulk silver value. A full conversion to bulk silver is not possible, since the organic materials decompose and evaporate during the sintering process [19], hence leaving a porous structure with a significant number of voids (Fig. 1).

Fig. 2 shows the remaining silver structure after the peel-off adhesion test for thermally sintered samples. The results show that upon peel-off almost all materials remain at the substrate. These samples can be assigned to category $4 \mathrm{~B} / 5 \mathrm{~B}$, according to the ASTM adhesion test standard (ASTM D-3359). In order to be comparable to thermal sintering, the conductivity achieved with the alternative sintering methods should be at least in the same range as can be achieved by thermal sintering and its adhesion to the polymer substrate should be in the category 4B-5B.

\subsection{Plasma sintering}

As a first selective sintering technique, plasma sintering was used. Inkjet printed silver nanoparticles on PI were sintered for 30,60 , 120 and $240 \mathrm{~min}$, respectively. It is known that plasma sintering progresses from top to bottom in the structures and the time for full sintering to take place depends on the thickness of the sample [19]. The sintering time was therefore varied to analyze the effect of plasma exposure time and progression of the sintering in the structure on both conductivity and mechanical performance. Thickness of the ink layer for plasma sintering was approximately $0.6 \mu \mathrm{m}$.
Fig. 3 shows SEM images of samples that were plasma sintered for 30 to $240 \mathrm{~min}$. A clear densification of the nanoparticles can be observed as well as a significant crack-formation within the track. Typically, crack formation takes place during the sintering process due to a material shrinkage and a quick removal of organic stabilizers [14,19].

The cross-section SEM images in Fig. 3 illustrate the top-to-bottom sintering behavior of plasma sintering as a function of sintering time. Image B in Fig. 3 shows a cross-section SEM image of a sample sintered for $30 \mathrm{~min}$ and it can be clearly seen that the top layer has sintered well, whereas the bottom layer shows unsintered nanoparticles. Image $C$ in Fig. 3 shows a cross-section SEM image of a sample that was sintered for $4 \mathrm{~h}$, which does not reveal a crust layer and hence the sintering process has completed through the structures down to the substrate. However, a difference in sintering process progression is clearly visible as the top layer has a much denser structure than the bottom. Therefore, it can be noted that both the initial sintering as well as the final densification start at the top of the structure and progress downward as sintering time is increased.

As a result of the progressing of the sintering process from top to bottom, plasma sintering is a rather slow sintering technique and, therefore, less suitable for roll-to-roll processing - unless very thin features are used. The resistivity, as shown in Table 1, was measured to decrease from $33.3 \mu \Omega \mathrm{cm}$ after $30 \mathrm{~min}$ to $15.0 \mu \Omega \mathrm{cm}$ after $240 \mathrm{~min}$. Additional sintering methods are required for high conductivity.

Another effect of the top-to-bottom sintering process is that if the sintering time is not sufficient to sinter the feature completely, the bottom part of the structure will remain unsintered. As that part determines the adhesion to the underlying substrate, adhesion problems may occur.

Fig. 4 shows the results of the peel-off adhesion test for plasma sintered test samples. Image A shows the remaining film at the substrate after the adhesion test for a sample that was plasma sintered for $30 \mathrm{~min}$. It can be seen that the samples were not completely sintered, indicated by the bluish appearance of the remaining non-conductive material at the surface. The top layer of the film that was removed by the tape (not shown here) was found to be silver colored and conductive, while the material left on the substrate was nonconductive. Therefore, the boundary between the sintered and unsintered layers determines the adhesion properties of the complete layer.

When using a sintering time of $4 \mathrm{~h}$ the adhesion has increased, see image $B$ in Fig. 4, which shows the remaining film at the substrate after the adhesion test. A clear difference in adhesion can be observed
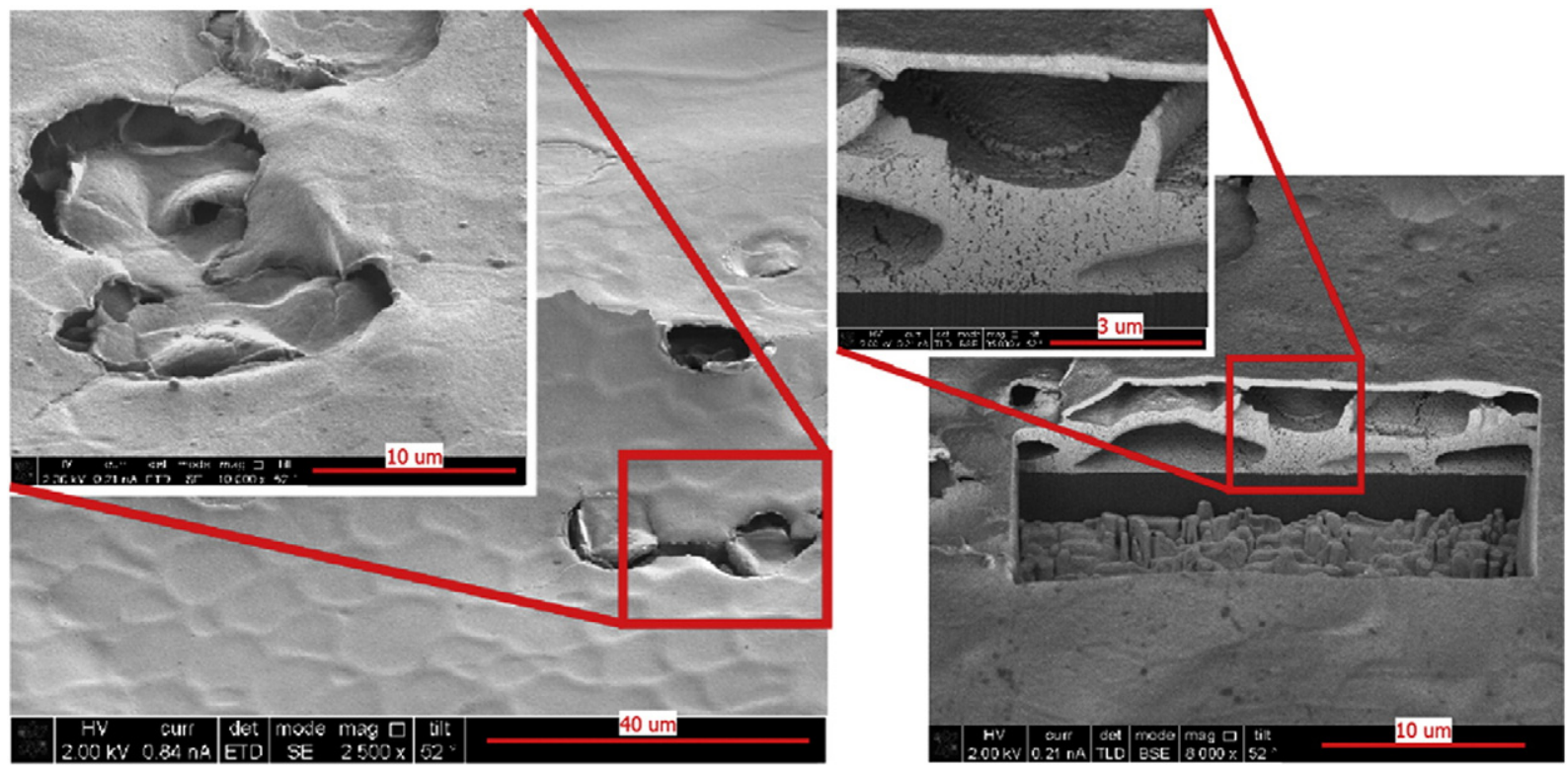

Fig. 5. Top-view and cross-section SEM images of laser sintered test samples, 


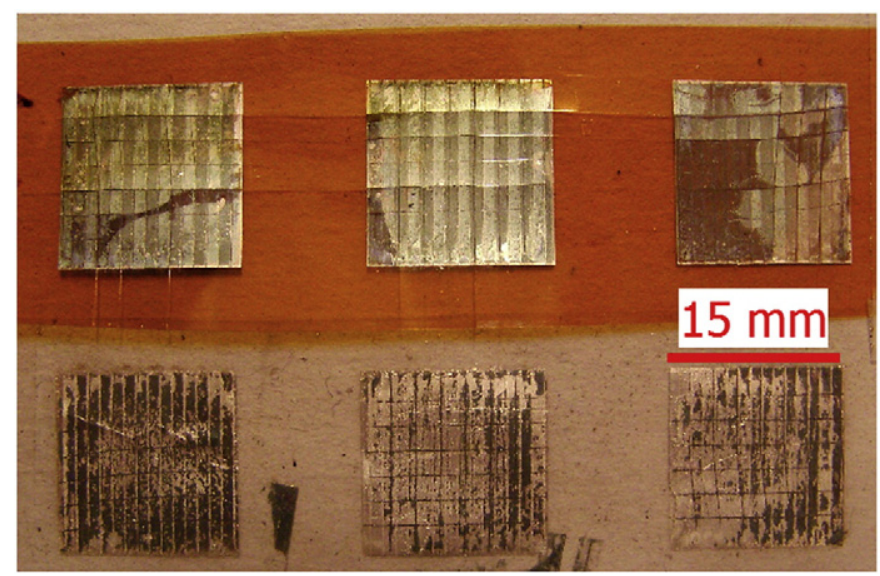

Fig. 6. Peel-off adhesion test sample for laser sintering on polyimide (top). The adhesion tape is shown at the bottom. The size of the peel-off adhesion test squares is $15 \mathrm{~mm}$ by $15 \mathrm{~mm}$.

compared to the sample that was sintered for $30 \mathrm{~min}$; the edges of the squares are now completely removed in the peel-off test, while the center of the structure has split in a similar fashion as the 30 min sintered sample. This would suggest that the edges are sintered well enough to form a uniform structure in the vertical direction, but the structure has not yet formed decent adhesion to the substrate. In the center of the structure the sintering has not yet progressed deep enough to form a uniform and mechanically strong structure in the vertical direction, leading to horizontal splitting of the structure. Although the remaining material in the center is silver colored, no conductivity was observed. Therefore, it can be concluded that for plasma sintering the process progression strongly depends on the planar geometry as well as the vertical thickness.

Despite that the image $\mathrm{C}$ in Fig. 3 indicates that the sintering process has started even in the bottom layer of the structure, it has not reached the point where a decent adhesion to the substrate material would be provided. And, as a result, the entire plasma sintered structure revealed a poor adhesion to the substrate. Sintering time has, therefore, a significant impact on the adhesion and the mechanical performance of the printed structure.

\subsection{Laser sintering}

As a third sintering technique, the inkjet printed lines were exposed to a laser source. The laser spot was smaller than the actual line width, which means that the sintering process requires several passes over the line to sinter the complete feature. Exposure of the laser beam to the substrate that was not covered by the ink is not harmful, as the substrate is transparent for this particular wavelength. Hence, the surrounding substrate is not heated, nor deformed by direct exposure to the laser. The substrate is, however, strongly affected by the indirect heat exposure, caused by the radiation that is absorbed by the ink and conducted to the substrate material [32].

Laser sintering locally heats up the area for a very short period of time, but the temperature may rise temporarily above the $\mathrm{Tg}$ of costeffective low-temperature polymer substrates, like PET and PEN. The exact process temperature depends on the used process parameters, such as sintering power and sweeping speed. In the experiments performed here, the process temperature was not measured, but may be estimated from experiments done on substrate materials with low thermal stability as well as from finite element simulations.

Several parameters, including optical power of the laser and scanning speed, were tested to find the optimal electrical conductivity and adhesion. It was noted that Harima NPS-J requires a high sintering power and a fast sintering speed in order to avoid local heating of the substrate. Tested process parameters for laser sintering and the resulting resistivity are summarized in Table 2 . When using a power of $38 \mathrm{~W}$ and a scanning speed of $80 \mathrm{~mm} / \mathrm{s}$ the lowest resistivity was $4.6 \mu \Omega \mathrm{cm}$, which equals $3 \times$ the bulk silver resistivity.

Fig. 5 shows top-view and cross-section SEM images of laser sintered silver nanoparticles on a polyimide substrate. It can be seen that the laser treatment converts the silver ink into a continuous and conductive feature. Large voids are clearly visible in both SEM images and the overall microstructure is highly porous. Large voids and general porosity may be formed due to a rapid decomposition and evaporation of the organic material from the ink.

In peel-off adhesion tests the laser sintered samples suffered from the same splitting behavior as the plasma sintered samples. Large voids and porosity reduce the mechanical integrity of the structure and the sintered crust of the structure was removed along with the adhesion tape, leaving behind a nonconductive silver structure. This particular case is not covered in the test standard but it is clear that the adhesion is insufficient for electronic applications and it can be assumed to be relative to category 0 . Peel-off adhesion test results can be seen in Fig. 6: on top is the polyimide substrate and the remaining part of the printed structure and on bottom the peeled off part of the structure that was removed along with the tape.

Large voids make the structure of the laser sintered sample poor and non-uniformal, see Fig. 5. However, this does not seem to affect the electrical performance significantly as a low resistivity can still be achieved. However, the effect on mechanical performance seems to be much more significant. The porous microstructure seen in Fig. 5 is far from optimal as holes within the structure provide easy crack propagation paths after initial failure has occurred, thus reducing the overall reliability.

\subsection{Photonic sintering}

Photonic sintering uses short pulses of an intense pulsed light source with a broad spectrum, ranging from the UV to the IR, to increase the temperature in the sample locally and rapidly. Due to the dark color of the silver nanoparticle ink and the transparency of the polymer foil, the light is only absorbed by the ink, hence creating a rapid temperature rise within the ink rather than in the polymer foil. The polymer foil is not damaged by the photonic sintering process directly, similar as for laser sintering. However, the indirect heating caused by the dissipated heat from the ink to the substrate may cause damage.

Several photonic sintering process parameters, including frequency, power level and total exposure time were varied in order to find optimal sintering conditions. The photonic sintering parameters are summarized in Table 3 and conductivity and adhesion results are presented in Table 4. The voltage pulse sent to the lamp is step function but due to delays in the reaction time of the lamp, the light pulse is a distorted bell function. The shape of the actual light pulse is presented in [33].

Table 3

Test parameters for photonic sintering test.

\begin{tabular}{|c|c|c|c|c|c|}
\hline Setting & Sintering time (s) & Sintering power $(\mathrm{kW})$ & Pulse length (ms) & Pulse frequency $(\mathrm{Hz})$ & Total energy $(\mathrm{kJ})$ \\
\hline 1 & 10 & 1 & 10 & 35 & 3.5 \\
\hline 2 & 10 & 1 & 10 & 50 & 5.0 \\
\hline 3 & 20 & 1 & 10 & 35 & 7.0 \\
\hline 4 & 40 & 1 & 10 & 35 & 14.0 \\
\hline
\end{tabular}


Table 4

Resistivity and peel-off adhesion test results for photonic sintering.

\begin{tabular}{lll}
\hline Setting & Resistivity $(\mu \Omega \mathrm{cm})$ & Adhesion $($ ASTM D-3359) \\
\hline 1 & $7.1-13.6$ & $4 \mathrm{~B} / 5 \mathrm{~B}$ \\
2 & $4.9-6.0$ & $4 \mathrm{~B} / 5 \mathrm{~B}$ \\
3 & $3.8-6.6$ & $4 \mathrm{~B} / 5 \mathrm{~B}$ \\
4 & $3.3-3.8$ & $4 \mathrm{~B} / 5 \mathrm{~B}$ \\
\hline
\end{tabular}

It can be seen that the achievable conductivity correlates with the total energy applied to the sample during flash sintering.

Fig. 7 shows SEM images of photonically sintered samples, clearly indicating that the sintering process has taken place, as large silver domain has formed. In Fig. 7, two photonic sintered samples with different process parameters are compared. The sample with a more porous microstructure has $5 \times$ the bulk silver resistivity, whereas the sample with a more uniformal microstructure has $2.5 \times$ the bulk silver resistivity. Therefore, it can be said that microstructure uniformity has a direct correlation to final conductivity.

The photonically sintered samples were then subjected to adhesion tests, the results of which are depicted in Fig. 8. The effect of the narrow focus area of the IPL beam can be clearly observed as a reflective stripe in the middle of the top image in Fig. 8. The lower left image shows the sintered structure after application of the adhesion tape, while the image on the lower right shows the adhesion tape used for peeling-off material from the adhesion squares. Photonic sintering technique has higher momentary sintering temperature than other techniques. The sintering temperature is high enough to cause minor thermal damage to the polyimide substrate, which can be observed in Fig. 8. Thermal damage is caused by the dissipated heat from the printed structure as mentioned earlier. By carefully tuning the pulse length, the flashing frequency, the sintering time as well as the intensity of the lamp, the sintering process can be adjusted so that substrate damage remains very limited.

It can be seen that the focus area of the photonic sintering equipment has a significant effect on the adhesion of the printed structure. In large-scale roll-to-roll fabrication, however, homogenous sintering can be realized by placing an array of flash lamps and by subsequently moving the foil with the printed ink patterns on top continuously through the focus line [34]. In the on-focus area the adhesion of the printed structure to the substrate is very good, either category $4 \mathrm{~B}$ or 5B according to the ASTM D3359-B adhesion test standard. Outside the focus area the silver ink has not sintered completely and, as a result, the adhesion is significantly poorer. A similar effect was observed when using plasma or laser sintering; the sintered silver top crust is removed in the adhesion test, i.e. the silver material remains on adhesive tape (see lower right part of Fig. 8) and the bottom part of the structure remains on the substrate after the adhesion test. This case does not fit into any category of the ASTM D3359-D test standard, but can be assumed that the results would belong into category 0 .

The parts of the adhesion squares that had not been directly in the focus area of the IPL beam were found to have not been sintered properly. Sufficient light has scattered so that the sintering process was initiated and the color of the ink has changed to resemble that of silver, but no conductivity was measured. Between the extremely wellconducting focus area of the IPL beam and the non-sintered edges of the squares the adhesion changed gradually from category 0 (at the edges) to $5 \mathrm{~B}$ (in the middle).

\section{Feasibility analysis}

The feasibility of all tested sintering methods is compared to evaluate which techniques are suitable for replacing conventional thermal sintering in large scale electronic manufacturing. The feasibility analysis is based on the conductivity and adhesion measurements as well as the suitability for large-scale roll-to-roll fabrication, the ability to achieve low sintering temperatures and control over the sintering process. An overview of this comparison can be seen in Table 5. Each sintering method is ranked in each criterion from a poor result $(---)$ to an excellent result $(+++)$, according to their capability to fulfill the specific requirements set for each criterion. From Table 5 it can be seen that none of the tested sintering techniques is perfect in every single aspect and, therefore, choosing a sintering technique is a matter of making compromises.

For "conductivity" criteria the sintering methods were ranked according to the lowest achieved resistivity. In this criteria both thermal sintering and photonic sintering achieve excellent grades as both techniques enable conductivities up to half of the bulk value for silver. Laser and plasma sintering achieved lower but still positive scores ("+ +" and "+"respectively) as their resistivities are higher but still adequate for some applications.

For "mechanical performance" criteria the sintering techniques were ranked according to the adhesion between the sintered structure and the polyimide substrate. Thermal sintering and photonic sintering showed good enough adhesion in the test to be ranked in the highest category in ASTM D-3359 standard and are therefore also given the highest rank in Table 6. In the adhesion testing both laser sintering and plasma sintering showed horizontal splitting and even though this scenario is not included in the ASTM D-3359 test standard it is
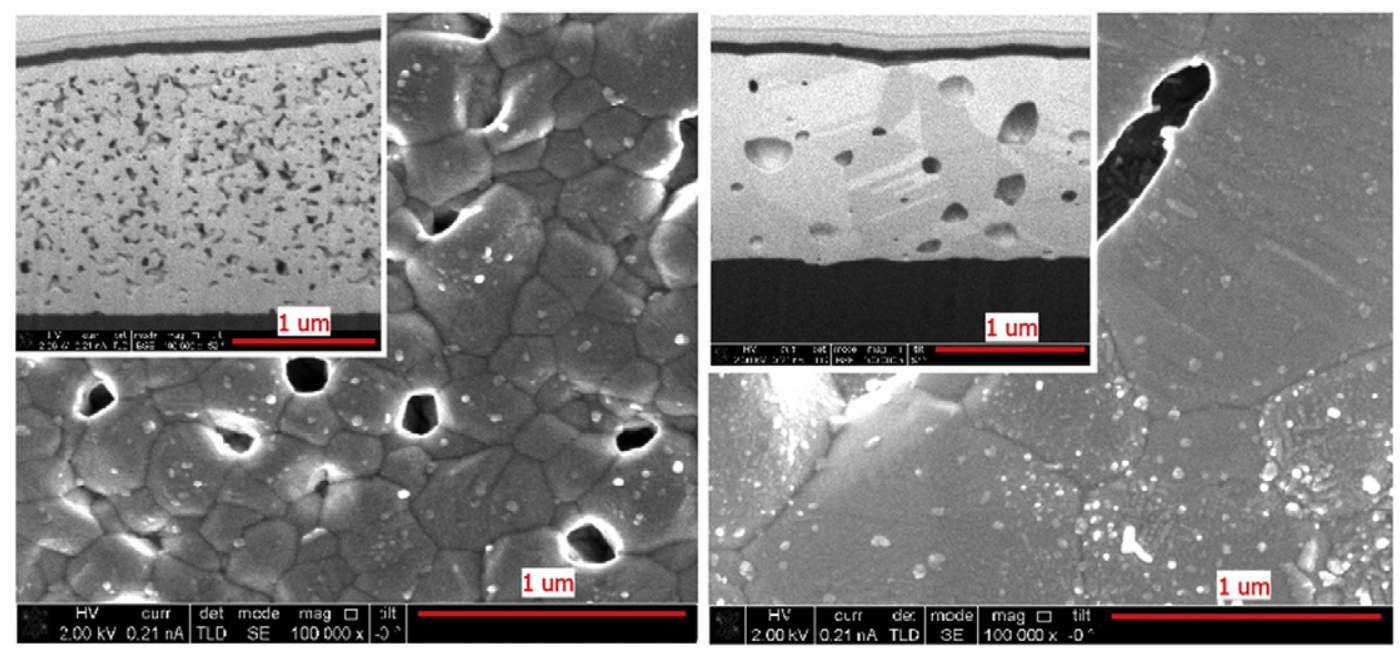

Fig. 7. Top-view and cross-section SEM images of photonic sintered samples. More porous structure is presented on the left side and more uniformal on the right. 


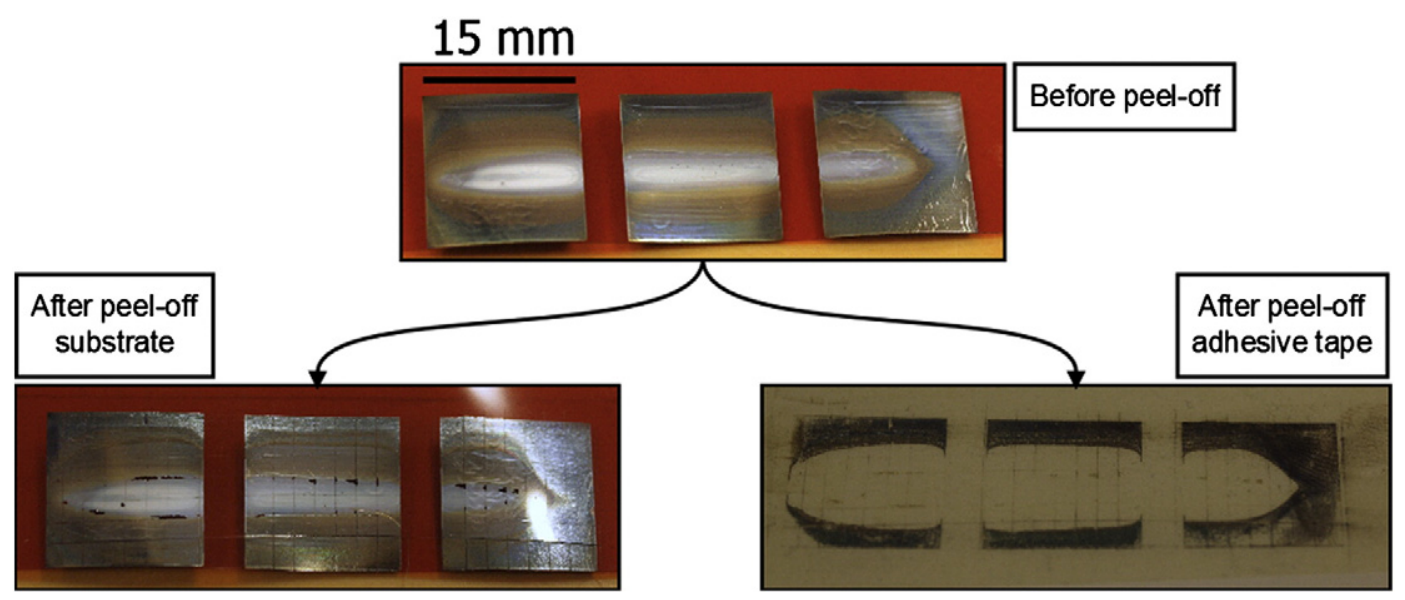

Fig. 8. Peel-off adhesion test results for photonically sintered test samples. The size of the peel-off adhesion test squares is $15 \mathrm{~mm}$ by $15 \mathrm{~mm}$.

clear that the mechanical performance of test samples sintered with these techniques is poor.

For "roll-to-roll compatibility" criteria the sintering techniques are ranked according to their suitability for the large scale manufacturing. Special attention is paid to sintering time and upscalability of the sintering equipment. Thermal sintering and plasma sintering have low scores (" - _ " and " - - - " respectively) due to their long sintering time which makes the roll-to-roll fabrication practically impossible. Plasma sintering is further hindered by the near-vacuum requirement which is inconvenient in a large scale. Both laser sintering and photonic sintering achieve high score in roll-to-roll compatibility ("+ +" and " +++ " respectively) as both techniques enable rapid sintering. Photonic sintering scores higher than laser sintering because of larger sintering area and thus easier upscaling for large scale fabrication.

For "temperature" criteria the sintering techniques are ranked according to the observed temperature requirements each sintering technique put on the substrate material. Thermal sintering is used as a traditional milestone to which the alternative sintering methods are compared to. Photonic sintering caused minor thermal damage to polyimide substrate which is successfully used with thermal sintering and therefore photonic sintering scores even lower than thermal sintering ("- - - " and "- - " respectively). Negative score ("-") is also given to laser sintering as some thermal damage occurs on when sintering on low temperature substrates. Plasma sintering is given a high score (" +++ ") as no thermal damage is observed even on low temperature polymer foils.

For "controllability" criteria the sintering techniques are ranked according to how well the sintering process can be controlled. Laser sintering is given the highest score (" +++ ") as the fully digital table and laser controlling enables very accurate controlling of the sintering power. This means that the sintering power can also be optimized according to the structure and substrate properties within a single structure. Photonic sintering enables some of the similar options but not nearly as accurately as laser sintering and therefore it is given a lower score ("+ +"). Thermal sintering and plasma sintering enable only very limited options for sintering parameter controlling and are therefore scored lower ("+" for both techniques).

\section{Conclusion}

As a summary, Table 6 shows the lowest resistivity values obtained with the four sintering technologies used in this study. It can be seen that all techniques reveal conductivity between $10 \%$ and $49 \%$ of the value of bulk silver, which is sufficient for printed electronic applications [24-26].

Excellent electrical and mechanical performance of the photonic sintered samples is most likely explained by the differences in the microstructures. Photonic sintered samples have a significantly better microstructure than plasma- or laser-sintered samples (compare Figs. 7, 3 and 5). Photonic sintering produces a more uniform and denser nanostructure than plasma- or laser-sintering, which has an effect on both electrical and mechanical performance.

From this study, photonic sintering can be identified as the most promising alternative sintering technique to replace conventional thermal sintering; high conductivity, good adhesion, short processing times and good scalabilities to a larger scale fabrication were revealed using this sintering technique. Careful tuning of the process parameters is, however, required in order to control the process temperature and to prevent substrate damage. Laser sintering has a potential for specific applications as the digital controlling enables an accurate and selective sintering process if the adhesion can be improved with further process development. For example, line structures can be selectively sintered in this way. As the slowest sintering technique tested here, plasma sintering may be useful in large-area sheet-to-sheet processing or as a pre-sintering method in combination with other sintering techniques [20].

\section{Acknowledgments}

The authors would like to thank the staff of Philips Innovation Services for the SEM imaging.

This work was supported by the Finnish Funding Agency for Technology and Innovation (Tekes) under Grant 40226/10.

MM is sponsored by the Academy of Finland under grant no. 251882.

Table 5

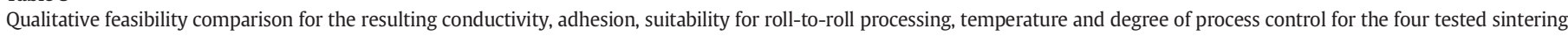
techniques.

\begin{tabular}{|c|c|c|c|c|c|}
\hline Sintering method & Conductivity & Mechanical performance & Roll-to-roll compatibility & Temperature & Controllability \\
\hline Thermal & +++ & +++ & -- & -- & + \\
\hline Plasma & + & --- & --- & +++ & + \\
\hline Laser & ++ & --- & ++ & - & +++ \\
\hline Photonic & +++ & +++ & +++ & --- & ++ \\
\hline
\end{tabular}


Table 6

Resistivity and peel-off adhesion test results for all of the tested sintering methods.

\begin{tabular}{lll}
\hline Sintering method & Resistivity $(\mu \Omega \mathrm{cm})$ & Adhesion $($ ASTM D-3359) \\
\hline Thermal & 3.6 & $4 \mathrm{~B} / 5 \mathrm{~B}$ \\
Plasma & $15.0-15.7$ & 0 \\
Laser & $4.6-6.2$ & 0 \\
Photonic & $3.3-3.8$ & $4 \mathrm{~B} / 5 \mathrm{~B}$ \\
\hline
\end{tabular}

The authors JN, MM and DL would like to thank Nokia, UPMkymmene, Premix and Meadville Aspocomp for financial support.

Furthermore, JN would like to thank Tuula ja Yrjö Neuvon rahasto and Nokia Foundation for personal research grants and Tampere University of Technology Presidents Doctoral Program for financial support.

The authors RA, JP and USS thank the European Community's Seventh Framework Programme (FP7/2007-2013) under grant agreement no. 248816 for financial support.

\section{References}

[1] Z. Yin, Y. Huang, N. Bu, X. Wang, Y. Xiong, Chin. Sci. Bull. 55 (2010) 3383.

[2] O.E. Association, Printed Electronics - Ready to go! http://www.vdma-webbox.tv/ english/filmdatabase/printed-electronics-ready-to-go.html2011.

[3] V. Subramanian, J.M.J. Fréchet, P.C. Chang, D.C. Huand, J.B. Lee, S.E. Molesa, A.R Murphy, D.R Redinger, S.K. Volkman, Proc. IEEE 93 (2005) 1330.

[4] C.C. Ho, K. Murata, D. Steingart, J.W. Evans, P.K. Wright, J. Micromech. Microeng. 19 (2009) 9.

[5] R. Jansen, M. Grätzel, J. Mater. Chem. 19 (2009) 5261.

[6] T. Sekitani, H. Nakajima, H. Maeda, T. Fukushima, T. Aida, K. Hata, T. Someya, Nat. Mater. 8 (2009) 494.

[7] J. Perelaer, P. Smith, D. Mager, D. Soltman, S. Volkman, V. Subramanian, J. Korvink, U.S. Schubert, J. Mater. Chem. 20 (2010) 8446.

[8] M. Singh, M. Haverinen, P. Dhagaat, G. Jabbour, Adv. Mater. 22 (2010) 673.

[9] E. Tekin, P. Smith, U.S. Schubert, Soft Matter 4 (2008) 703.

[10] J. Miettinen, V. Pekkanen, K. Kaija, P.M.J. Mäntysalo, M. Mäntysalo, J. Niittynen, J. Pekkanen, T. Saviauk, R. Rönkkä, Microelectron. J. 39 (2008) 1740.
[11] K. Kaija, V. Pekkanen, M. Mäntysalo, P. Mansikkamäki, Proc. SMTA International Conference, Orlando, FL, USA, 2008, p. 1.

[12] S.K. Volkman, S. Yin, T. Bakhishev, K. Puntambekar, V. Subramanian, M.F. Toney, Chem. Mater. 23 (2011) 4634

[13] T.H.J. van Osch, J. Perelaer, A.W.M. de Laat, U. Schubert, Adv. Mater. 20 (2008) 343.

[14] J. Perelaer, A.W.M. de Laat, C.E. Hendriks, U.S. Schubert, J. Mater. Chem. 18 (2008) 3209.

[15] H.-H. Lee, K.-S. Chou, K.-C. Huang, Nanotechnology 16 (2005) 10.

[16] S. Magdassi, M. Grouchko, O. Berezin, A. Kamyshny, ACS Nano 4 (2010) 1943.

[17] M. Allen, J. Leppäniemi, M. Vilkman, A. Alastalo, T. Mattila, Nanotechnology 21 (2010) 47.

[18] T. Kumpulainen, J. Pekkanen, J. Valkama, J. Laakso, R. Tuokko, M. Mäntysalo, Opt. Laser Technol. 43 (2011) 570.

[19] I. Reinhold, C.E. Hendriks, R. Eckardt, J.M. Kranenburg, J. Perelaer, R. Baumann, U.S. Schubert, J. Mater. Chem. 19 (2009) 3384

[20] J. Perelaer, M. Klokkenburg, C.E. Hendriks, U.S. Schubert, Adv. Mater. 21 (2009) 4830.

[21] M. Allen, M. Aronniemi, T. Mattila, A. Alastalo, K. Ojanperä, M. Suhonen, H. Seppä, Nanotechnology 19 (2008) 17.

[22] H.-S. Kim, S. Dhage, D.-E. Shim, H.T. Hahn, Appl. Phys. A 97 (2009) 791.

[23] M. Allen, Ph.D. Thesis, Aalto University, Finland, 2011.

[24] V. Pynttäri, E. Halonen, H. Sillanpää, M. Mäntysalo, R. Mäkinen, IEEE Antennas Wirel. Propag. Lett. 11 (2012) 188.

[25] V. Pekkanen, M. Mäntysalo, P. Mansikkamäki, Proc. IMAPS 40th International Symposium on Microelectronics, San Jose, CA, USA, 2007, p. 1076.

[26] V. Pynttäri, R. Mäkinen, J. Lilja, V. Pekkanen, P. Mansikkamäki, M. Kivikoski, Proc. 12th IEEE Workshop on Signal Propagation on Interconnects (SPI), Avignon, France, 2008.

[27] M. Mäntysalo, P. Mansikkamäki, AEU Int. J. Electron. Commun. 63 (2009) 31.

[28] E. Halonen, V. Pynttäri, J. Lilja, H. Sillanpää, M. Mäntysalo, J. Heikkinen, R. Mäkinen, T. Kaija, P. Salonen, Microelectron. Eng. 88 (2011) 2970.

[29] M. Leenen, V. Arning, H. Thiem, J. Steiger, R. Anselmann, Phys. Status Solidi A 206 (2009) 588

[30] M. Klokkenburg, G.T. van Heck, E. Rubingh, T. van Lammeren and H. A. J. M. Andriessen, PCT Eur. Appl. Patent, 2010.

[31] ASTM Standard D-3359-09e2 Test Methods for Measuring Adhesion by Tape Test, 2009.

[32] J. Chung, S. Ko, N. Bieri, C. Grigoropoulos, D. Poulikakos, Appl. Phys. Lett. 84 (2004) 801.

[33] R. Abbel, T. van Lammaren, R. Hendriks, J. Ploegmakers, E.J. Rubingh, E.R. Meinders, W.A. Groen, MRS Commun. 2 (2012) 145.

[34] J. Perelaer, R. Abbel, S. Wünscher, R. Jani, T. van Lammeren, U.S. Schubert, Adv. Mater. 24 (2012) 2620. 